\title{
Critical Operations Selecting Method
}

\author{
LI Qiuying ${ }^{1,2, a}$, LUO Lei Li,b $^{1,2}$ \\ ${ }^{1}$ School of Reliability and Systems Engineering, Beihang University, China \\ ${ }^{2}$ Science \& Technology on Reliability \& Environmental Engineering Laboratory, China \\ ali_qiuying@buaa.edu.cn, ${ }^{\mathrm{b}}$ leicherry@163.com
}

Keywords: critical operations, software reliability testing, safety testing, analytic hierarchy process.

\begin{abstract}
It is very important to select critical operations in software reliability testing and software safety testing. In this paper, a framework for selecting critical operations based on Analytic Hierarchy Process (AHP) is proposed. The hierarchies of goals, criteria and alternative programs are introduced separately. A preliminary application is practiced to show the method's application process and demonstrate the feasibility of this method, which can be taken as a reference for critical operations' identification in engineering application.
\end{abstract}

\section{Introduction}

For high reliability and safety-critical software, it usually takes a long time or huge amount of test cases to do software reliability testing and safety testing by conventional methods, which leads to the fact that the conventional methods cannot be put into use for accurately predicting failure rates of a very high reliability or safety-critical software [1]. To solve this problem, importance sampling (IS) method is applied in software reliability testing and software safety testing [2,3]. An accelerated software testing method is put forward based on the strengthened operational profile (OP) which is constructed on Musa's OP [4] and its foundation is to identify critical operations reasonably and effectively [5].

Now critical operations' identification can only be referred by similar software [6]. Reference [3] researches on software safety testing using fault tree analysis (FTA) method to identify hazard operations. Through FTA to find the basic events which may cause the incident, find out operations related to these events, then construct a correlative risk profile [7]. This approach is considerable, but for high reliability software risk of accidents is only part of factors to be considered. AHP [8] is a multi-criteria decision-making technique that has been widely used to solve complex decision problems [9]. Moreover AHP has been widely applied in engineering, industry, manufacturing and so on [10]. This paper puts forward a new framework which uses AHP to identify critical operations, and gives a systematic and comprehensive guidance to application.

\section{Critical Operations selecting method based on AHP}

After Musa's OP is constructed, the basic characteristic of each operation has been identified. However, the influence degree of each operation on reliability is uncertain. It is related to the occurrence probability, the failure consequence and other factors. These factors have different weight, therefore a comparative analysis of all factors is very difficult. AHP is a way to analyze quantitative problem qualitatively and provide a simple approach for decision-making.

\section{AHP method}

The basic principle of AHP is to convert complex problem into several levels and factors. Then every two factors contrast with each other to get the weights separately. From analysis and calculation through low to high layers, each program's weight is obtained for the overall goal. The largest weight of the program is the optimal solution [10].

The Procedure to Identify Critical Operations

- Construct a multi-hierarchy structure 
Evaluation criteria mainly include: i) Operation’s probability; ii) Mission-critical level; iii) Consequence of operation's failure; iv) domain of operation. The hierarchy model is shown in Fig. 1.

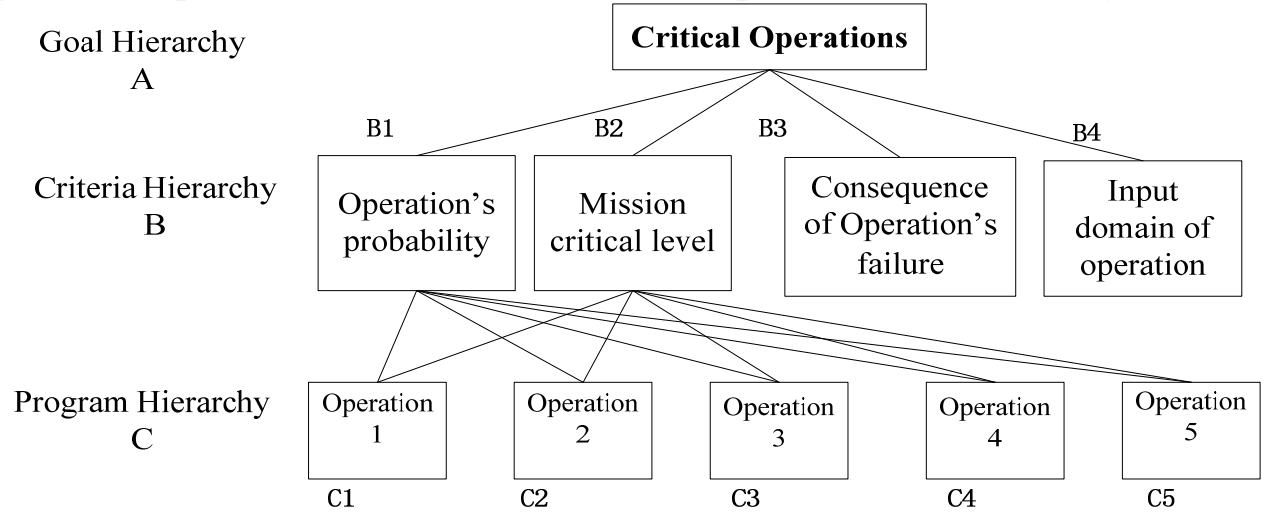

Figure 1 Multi-hierarchy model

- Construct the comparison matrix of criteria hierarchy

Table 1 The definition of 1-9 Scale

\begin{tabular}{cc}
\hline Relative importance $a_{i j}$ & Definition \\
\hline 1 & The goal $i$ and $j$ are equally important \\
5 & The goal $i$ is a little more important than $j$ \\
7 & The goal $i$ is more important than $j$ \\
9 & The goal $i$ is much more important than $j$ \\
$2,4,6,8$ & The goal $i$ is more important than $j$ absolutely \\
\hline
\end{tabular}

1-9 scale is used to determine the matrix, which takes the two programs of the relative importance as elements shown in Table 1. The comparison matrix of criteria hierarchy is given as follows:

\begin{tabular}{c|cccc}
$A$ & $B 1$ & $B 2$ & $B 3$ & $B 4$ \\
\hline$B 1$ & 1 & 3 & 5 & 7 \\
$B 2$ & $1 / 3$ & 1 & $1 / 2$ & 4 \\
$B 3$ & $1 / 5$ & 2 & 1 & 5 \\
$B 4$ & $1 / 7$ & $1 / 4$ & $1 / 5$ & 1
\end{tabular}

\section{- Construct the comparison matrix of operation hierarchy based on each criterion}

If $n$ operations are to be decided, four $n \times n$ matrixes $(F)$ should be built, representing the importance weights of all operations based on these four criteria.

\section{- Normalize the comparison matrix and calculate the weights}

Firstly, use normalization method to calculate each matrix line, then convert $(F)$ to $\left(F^{\prime}\right)$. Secondly, the sum of each matrix row should be calculated. Then determine weights for all the sums pf row. Here take comparison matrix of criteria hierarchy for example shown in Table 2.

Table 2 Weights of the criteria hierarchy

\begin{tabular}{lrrrccc}
\hline A & B1 & B2 & B3 & B4 & Sum of row & Weight \\
\hline B1 & 0.5967 & 0.48 & 0.7463 & 0.4118 & 2.2348 & 0.5587 \\
B2 & 0.1989 & 0.16 & 0.0746 & 0.2353 & 0.6688 & 0.1672 \\
B3 & 0.1193 & 0.32 & 0.1493 & 0.2941 & 0.8827 & 0.220675 \\
B4 & 0.0851 & 0.04 & 0.0298 & 0.0588 & 0.2137 & 0.053425 \\
Sum & 1 & 1 & 1 & 1 & 4 & 1 \\
\hline
\end{tabular}

\section{- Consistency analysis}

The elements of comparison matrix have three mathematical properties. $a_{i i}=1, a_{i j}=1 / a_{j i}, a_{i j}=a_{i k} \cdot a_{k j}$. Comparison matrix is generally estimated based on knowledge and experience of makers. As decision-makers do not have accurate estimates, they may fail to meet the need of the third property. Since consistency analysis is necessary, the average random consistency ratio $C R$ should be calculated.

When $C R<0.1$, it means that comparison matrix conforms to consistency. Then we have 


$$
C I=\frac{\lambda_{\max }-n}{n-1}
$$

where

$$
\lambda_{\max }=\frac{1}{n} \sum \frac{(F W)_{i}}{w_{i}}
$$

Then we have

$$
C R=\frac{C I}{R I}
$$

- The final selection results

Weights vector of criteria hierarchy is given as follows.

$$
\begin{array}{lll}
B_{41}=\left[\begin{array}{llll}
0.5587 & 0.1672 & 0.220675 & 0.053425
\end{array}\right]^{T} \\
\text { Then we have } & V_{n 1}=\left[C_{i j}\right]_{n 4} B_{41}
\end{array}
$$

\begin{tabular}{|c|c|c|c|c|c|c|}
\hline \multicolumn{2}{|c|}{ Criteria } & $\begin{array}{l}\text { Operation's } \\
\text { probability }\end{array}$ & $\begin{array}{c}\text { Mission-critical } \\
\text { level }\end{array}$ & $\begin{array}{l}\text { Consequence } \\
\text { s of } \\
\text { operation's } \\
\text { failure }\end{array}$ & $\begin{array}{c}\text { Input } \\
\text { domain of } \\
\text { operation }\end{array}$ & $\begin{array}{c}\text { Total } \\
\text { weights of } \\
\text { all the } \\
\text { operation }\end{array}$ \\
\hline \multicolumn{2}{|c|}{ Weights of the criteria hierarchy } & 0.5587 & 0.1672 & 0.220675 & 0.053425 & $\mathrm{~s}$ \\
\hline \multirow{4}{*}{$\begin{array}{l}\text { Weights of } \\
\text { programs } \\
\text { hierarchy for the } \\
\text { four Criteria }\end{array}$} & Operation 1 & & \multirow{4}{*}{\multicolumn{2}{|c|}{$\left[C_{i j}\right]_{n 4}$}} & & \multirow{4}{*}{$V_{n 1}$} \\
\hline & Operation 2 & & & & & \\
\hline & ...... & & & & & \\
\hline & Operation $n$ & & & & & \\
\hline
\end{tabular}

The last matrix $V_{41}$ is shown in Table 3, which represents total weights of all the operations. Sort the operations by total weights, we can choose the front one-third or half operations as critical operations, according to the accelerated degree we need.

Table 3 Total weights

\section{Application research}

Here we take a control software for example. As the actual OP is complicated, we analyse only a small part of operations in the Musa's OP and show the process to select critical operations.

List the operations with their probability as programs hierarchy

Operations should be analyzed are: C1: Self-test (0.021); C2: Data-loading (0.057); C3: Aerial

\begin{tabular}{|c|c|c|c|c|c|c|c|c|c|c|c|c|c|c|c|c|c|c|c|c|c|c|c|}
\hline$B 1$ & $C 1$ & $C 2$ & C3 & $C 4$ & C5 & $B 2$ & $C 1$ & $C 2$ & C3 & $C 4$ & C5 & B3 & $C 1$ & $C 2$ & C3 & $C 4$ & C5 & B4 & $C 1$ & C2 & C3 & $C 4$ & C5 \\
\hline$C 1$ & 1 & $1 / 3$ & $1 / 6$ & $1 / 8$ & $1 / 2$ & $\overline{C 1}$ & 1 & 2 & $1 / 7$ & $1 / 8$ & 2 & $\overline{C 1}$ & 1 & 5 & 3 & 3 & 5 & $\overline{C 1}$ & 1 & $1 / 7$ & $1 / 3$ & $1 / 3$ & $\overline{1 / 2}$ \\
\hline & 3 & 1 & $1 / 5$ & $1 / 6$ & 2 & $C 2$ & $1 / 2$ & 1 & $1 / 7$ & $1 / 8$ & 1 & $C 2$ & $1 / 5$ & 1 & 5 & 5 & 6 & $C 2$ & 7 & 1 & 5 & 5 & 6 \\
\hline & 6 & 5 & 1 & $1 / 2$ & 7 & $=\mathrm{E} C 3$ & 7 & 7 & 1 & 1 & 7 & C3 & $1 / 3$ & $1 / 5$ & 1 & 1 & 7 & $=C 3$ & 3 & $1 / 5$ & 1 & $1 / 2$ & 2 \\
\hline$C 4$ & 8 & 6 & 2 & 1 & 7 & $C 4$ & 8 & 8 & 1 & 1 & 7 & $C 4$ & $1 / 3$ & $1 / 5$ & 1 & 1 & 7 & $C 4$ & 3 & $1 / 5$ & 2 & 1 & 5 \\
\hline$C 5$ & 2 & $1 / 2$ & $1 / 7$ & $1 / 7$ & 1 & C5 & $1 / 2$ & 1 & $1 / 7$ & $1 / 7$ & 1 & C5 & $1 / 5$ & $1 / 6$ & $1 / 7$ & $1 / 7$ & 1 & $C 5$ & 2 & $1 / 6$ & $1 / 2$ & $1 / 5$ & 1 \\
\hline
\end{tabular}
cannon attack (0.000134); C4: Missile attack (0.000089); C5 Patrol (0.048).

If no any other criterion is to be added, the weights of criteria hierarchy can be chosen as the values of $B_{41}$, so $B_{41}=\left[\begin{array}{llll}0.5587 & 0.1672 & 0.220675 & 0.053425\end{array}\right]^{T}$.

\section{Construct the comparison matrix}

\section{Calculate the weights vector}

$$
W_{1}=\left[\begin{array}{c}
0.04334 \\
0.097099 \\
0.326584 \\
0.470828 \\
0.062149
\end{array}\right] \quad W_{2}=\left[\begin{array}{l}
0.077261 \\
0.049741 \\
0.39973 \\
0.422021 \\
0.051246
\end{array}\right] \quad W_{3}=\left[\begin{array}{l}
0.405852 \\
0.293151 \\
0.131609 \\
0.131609 \\
0.037779
\end{array}\right] \quad W_{4}=\left[\begin{array}{c}
0.053084 \\
0.542616 \\
0.12515 \\
0.203579 \\
0.075239
\end{array}\right]
$$

\section{Consistency analysis}

For the first matrix, we can have the follows: 


$$
\lambda_{\max }=\frac{1}{5}\left(\frac{0.2201}{0.04334}+\frac{0.4954}{0.097099}+\frac{1.7426}{0.326584}+\frac{2.4881}{0.470828}+\frac{0.3114}{0.062149}\right)=5.162 .
$$

As $n$ is 5 , so $R I=1.11, C I_{1}=\frac{5.162-5}{5-1}=0.04, C R_{1}=\frac{0.04}{1.11}=0.036<0.1$. Thus matrix $F_{1}$ has good consistency. In the same way we can get $C R_{2}=0.0176, C R_{3}=0.023, C R_{4}=0.011$.

\section{Overall weight}

$$
V_{51}=\left[\begin{array}{cccc}
0.04334 & 0.077261 & 0.405852 & 0.053084 \\
0.097099 & 0.049741 & 0.293151 & 0.542616 \\
0.326584 & 0.39973 & 0.131609 & 0.12515 \\
0.470828 & 0.422021 & 0.131609 & 0.203579 \\
0.062149 & 0.051246 & 0.037779 & 0.075239
\end{array}\right]\left[\begin{array}{c}
0.5587 \\
0.1672 \\
0.220675 \\
0.053425
\end{array}\right]=\left[\begin{array}{c}
0.12953 \\
0.156246 \\
0.285026 \\
0.373533 \\
0.055648
\end{array}\right]
$$

According to $V_{51}$, the weights orders are: C4:Missile attack, C3:Aerial cannon attack, C2:Data-loading, C1:Self-test, C5:Patrol. So we can conclude that the critical operations are C4 and C3.

\section{Conclusion}

It is very important to identify critical operations properly in software reliability testing and safety testing. A method based on AHP is put forward to identify critical operations taking operational profile as an example in this paper. All the processes of this method are given and some results are shown by an example.

\section{References}

[1] R W. Butler and G. B. Finelli. The Infeasibility of Quantifying the Reliability of Life-Critical Real-Time Software. IEEE Transactions on Software Engineering, 1993, 19(1):3-12.

[2] M. Hecht and H. Hecht. Use of importance sampling and related techniques to measure very high reliability software. Aerospace Conference Proceedings 2000, 533-546.

[3] Z.D. QIN, X.Q. LIU, H.Y. WANG and J.J. LE. Software safety growth testing method based on correlative risk profile. Systems Engineering and Electronics,2009, 31(5)(in Chinese).

[4] Q.Y. LI, X. Li, J.WANG and L. LUO. Study on the accelerated software reliability demonstration testing for high reliability software based on strengthened operational profile. Proceedings of international conference on Computer Technology and Development (ICCTD), 2010.

[5] S. Alam, H. Chen and K. Ehrlieh. Assessing software reliability performance under highly critical but infrequent event occurrences. Proceedings of The Eighth International Symposium On Software Reliability Engineering, 1997, 294-307.

[6] D. Tang, M. Hecht, J. Miller, L. Czekalski and J. Handal. MEADEP and Its Applications in Evaluating Dependability for Air Traffic Control Systems, Proceedings of the Annual Reliability and Maintainability Symposium, Anaheim, CA, Jan. 19-22, 1998.

[7] Z.D.QIN. Study on Testing and Evaluation Theory of Highly Credible Software, Ph.D thesis, 2005(in Chinese).

[8] T.L. Saaty. Decision Making with Dependence and Feedback. Analytic Network Process, Pittsburgh: RWS, 2001.

[9] L.B. Yang and Y.Y. Gao. Fuzzy mathematic elements and application. GuangZhou: South China University of Technology Press, 2002.

[10] O.S.Vaidya and S. Kumar. Analytic hierarchy process: An overview of applications, European Journal of Operational Research, 2006, 169(1):1-29. 